\title{
Prioritizing the capabilities of unmanned underwater vehicles necessary for ISR based on the Analytic Hierarchy Process
}

\author{
Gwuihyeon Park ${ }^{*}$ Taehyun Lee ${ }^{* *}$ Chunghwan Lee ${ }^{* * *} \cdot J_{1 n h o}$ Lee $^{* * * *}$
}

\footnotetext{
The need for unmanned underwater vehicles (UUVs) has received significant attention given the current security situation in the Korean Peninsula. UUVs are used in the military to perform five key missions: intelligence, surveillance, and reconnaissance (ISR), mine countermeasures, anti-submarine warfare, inspection/identification, and oceanography. In this study, we analyze the standards of evaluation of the characteristics and capabilities of UUVs that are necessary for ISR missions, which are most important from among the above mentioned ones. First, we establish 5 main criteria and 15 sub-criteria by consulting a group of experts. Next, we use the analytic hierarchy process (AHP) for these criteria. It computes the relative weights of the criteria based on

() (1) This work is licensed under a Creative Commons Attribution 4.0 International License.

* (First Author) Korea Naval Academy, Department of National Defense Management, Student, asa975@naver.com

** (Co-Author) Korea Naval Academy, Department of National Defense Management, Student, taelee456@gmail.com

*** (Co-Author) Korea Naval Academy, Department of National Defense Management, Student, leech3348@naver.com **** (Corresponding Author) Korea Naval Academy, Department of National Defense Management, Professor, jinho7956@gmail.com
} 
pairwise comparisons, in order to evaluate the relative importance and priorities of the criteria we established, by selecting another group of 51 experts for the evaluation of the pairwise comparisons among the criteria. Consequentially, the importance associated with performance and ability of the ISR tends to be relatively high while the one associated with cost and economy for operating the UUVs appears to be low. These results may be useful in analyzing required capabilities in a more objective and concrete manner while procuring UUVs in the future.

Keywords : analytic hierarchy process (AHP), unmanned underwater vehicle, intelligence, surveillance and reconnaissance, required capability 
Prioritizing the capabilities of unmanned ... / Gwuihyeon Park • Taehyun Lee $\cdot$ Chunghwan Lee $\cdot$ Jinho Lee 3

\section{I. 서론}

\section{1 연구배경 및 목적}

첨단 과학기술의 발전과 미래 전장 환경의 변화는 전쟁수행 형태에도 변화를 일으키고 있다. 이 러한 추세에 맞추어 선진국을 중심으로 각국은 유인체계를 대체 또는 보완하는 형태로 무인체계에 대한 연구개발을 활발하게 진행하고 있다. Choi et al.(2012)는 무인체계의 필요성에 대하여 C4ISR(Command, Control, Communications, Computers, Intelligence, Surveillance and Reconnaissance) 프로세스를 통해 설명하였는데, C4ISR 사이클에서 무인체계는 정보수집 및 평가/ 확인을 위한 센서 역할, 네트워크 내에서 플랫폼과 센서 및 도구 간 통신 중계 역할, 공격을 위한 수단으로서의 역할 등을 담당할 수 있다. 또한, 무인체계는 대량살상과 파괴를 지양하고 비대칭 저비용 전력으로 군사력 우위를 유지하고 전쟁을 억제하는데 기여할 것으로 기대된다.

2010년 천안함 사건 이후 대한민국 해군은 수중에서 무인잠수정(Unmanned Underwater Vehicle, 이하 UUV)의 필요성을 절감한 결과, 2011년 11월에 최초의 군사용 UUV 개발을 목적으로 ‘수중탐색용 자율 무인잠수정 개발' 사업이 착수된 이후 현재까지 UUV에 관한 연구개발 및 사업이 활발히 진행되고 있다(Choi et al., 2012). 또한, 최근 한반도에서 직접적인 위협이 되고 있는 북한 잠수함발사탄도미사일(SLBM: Submarine-Launched Ballistic Missile)과 같은 비대칭 전력에 대응 할 수 있는 새로운 대잠 무기체계의 필요성에 따라 혁신적인 무기체계인 UUV의 개발 및 운용이 필수적으로 요구되는 상황이다(Lee, 2017). UUV는 인명피해 감소, 경제적 효율성, 전투 수행개념 및 무기체계 발전추세에 부합하기 위한 측면에서 그 역할과 중요성이 점점 증대되고 있으며(Lee, 2017), 미래 전투수행 개념에도 부합할 수 있는 무기체계로서 원거리 수중 감시정찰 등의 임무를 지속적이고 효과적으로 수행할 수 있는 수단으로도 활용될 수 있다(Kang et al., 2019).

최근의 안보상황과 그에 따른 UUV의 필요성이 충분히 부각되고 있는 것은 주지하고 있는 사실 이다. 그러나 이러한 필요성에 부합하는 UUV의 개발을 위해서는 보다 구체적인 선행연구가 요구 된다. 특히 UUV는 유인잠수정에 비해 소형이며 탑재장비 또한 제한되므로, 운용되는 목적에 따라 $\mathrm{UUV}$ 의 탐재장비 및 요구성능 등이 달라질 수 있으며, 이는 특히 군사작전을 위한 UUV에 더욱 민감하게 받아들여질 수 있는 요소이다. 현재 군사 분야에서 UUV의 핵심 임무는 정립되어 있으나, 각 임무별 고려요소와 요구성능에 대한 기준을 정량적으로 도출한 연구는 미흡한 실정이다. 따라서 본 연구에서는 UUV가 수행하게 될 핵심 임무를 효과적으로 수행하기 위해 요구되는 능력을 제시 하고 이를 평가하는 기준을 제시하고자 한다. 


\section{2 선행연구 고찰}

$\mathrm{UUV}$ 에 대한 필요성과 중요도의 증가를 반영하듯 국내 - 외에서도 많은 연구가 이루어져 왔다 (Lee, 2019). 특히 군사 분야에서 미국은 '2004 UUV Master Plan(US Navy, 2004)'을 통해 UUV의 다양한 임무 수행에 필요한 기술과 로드맵을 정의하였고 미 해군에 필요한 UUV의 핵심임무 9가지 를 제시하였는데 그 중 정보/감시/정찰, 대기뢰전, 대잠전, 조사/식별, 해양탐사 등 5 개의 임무를 중 요도가 가장 높은 것으로 평가하였다. 또한, 이를 바탕으로 Button et al.(2009)은 무인잠수정의 임 무를 추가적으로 분석하여 대기뢰전, 감시센서 배치, 항만감시, 해양탐사, 수중 기반시설 감시, 대잠 전 추적, 조사/식별 등 7가지의 추천 임무를 제시하였으며, Allard \& Shahbazian(2014)는 무인잠수 정을 통한 정보수집 활동의 용이성을 위한 데이터 통합 및 상호운용성에 대하여 제시하였다. 보다 최근인 2018년 발표된 미 해군의 자율무인잠수정 비전에 따르면, 미 해군은 UUV를 소형, 중형, 대 형 크기별로 구분하여 주력 플랫폼을 정의하였으며, 1 단계는 정보감시정찰, 2 단계에서는 대잠전과 대기뢰전을 포함하는 등 단계적인 비전을 제시하였다(Lee, 2020). Yao et al.(2019)은 정보감시정찰, 대잠전, 대기뢰전 등의 주요 임무를 수행함에 있어 발생 가능한 위협을 평가하기 위하여 동적 베이 지안 네트워크 모델을 제시하였다.

한편 국내에서는 Hwang et al.(2011)이 UUV의 여러 임무 중 기뢰 탐색 임무를 위한 제원 및 필 요 요구성능을 결정할 수 있는 시뮬레이터에 관해 연구하였고, Lee(2017)는 대잠 임무를 수행하는 UUV 개발 시 고려되어야 하는 요소를 바탕으로 운용개념 및 발전방향에 대해 제시하였다. 또한 Kang et al.(2019)은 UUV 운용개념을 기반으로 대잠정찰을 위한 UUV 운용 시나리오를 제시하였 고, Shin et al.(2020)은 대잠전 운용개념 중 가장 주요한 임무로 판단된 위험제어 시나리오를 기반 으로 시뮬레이터를 개발하였다. UUV의 운용개념에 관한 연구와 더불어 공학/기술적 관점에서의 연구 또한 진행되었다. Lee \& Yoon(2011)는 UUV의 정확한 위치추정을 위해 다중센서 융합을 기 반으로 UUV의 위치추정 방법을 개선하는 알고리즘을 연구하였다. Yoo et al.(2016)은 수중에서 GPS 정보를 사용할 수 없는 UUV의 단점을 보완하기 위한 탐색 UUV용 HILS(Hardware in the loop simulation) 시스템에 대한 연구를 진행하였다.

본 논문의 구성은 다음과 같다. 먼저 2장에서 $\mathrm{AHP}$ 기법에 대한 요약을 바탕으로, 3장에서는 감 시 - 정찰 목적의 UUV가 갖추어야 할 요구성능에 대한 평가기준을 선정하였고, 4장에서 설문조사 결과 분석 및 $\mathrm{AHP}$ 기법 활용 결과를 분석하였다. 마지막으로 5장에서는 결론과 $\mathrm{AHP}$ 기법 활용 결과에 근거한 감시 - 정찰용 UUV 개발이 나아가야 할 방향을 제시하였다. 
Prioritizing the capabilities of unmanned ... / Gwuihyeon Park • Taehyun Lee $\cdot$ Chunghwan Lee $\cdot$ Jinho Lee 5

\section{П. AHP 기법}

본 연구는 UUV의 핵심 임무 중에서도 가장 중요도가 높은 것으로 평가되는 감시 - 정찰 임무에 대하여, UUV가 갖추어야 할 객관적이고 구체적인 요구성능 및 평가기준을 제시하는데 그 목적이 있다. 이를 위하여, 먼저 해당 분야 전문가와의 면담 및 자료조사를 통해 감시·정찰용 UUV의 특 성을 도출하였으며, 세부적으로 5가지 대기준과 각각의 대기준별 3 가지 소기준을 설정함으로서 총 5 가지의 대기준 및 15 가지의 소기준으로 구분하였다. 다음으로 각 기준에 대한 우선순위를 도출하 기 위하여 전문가 집단의 설문을 실시하였고, $\mathrm{AHP}$ (Analytic Hierarchy Process) 기법을 이용하여 특성간의 우선순위를 도출하였다.

\section{1 개요}

$\mathrm{AHP}$ 기법은 다속성 의사결정방법 중 하나로 Saaty(1980)에 의해 개발된 방식이다. 다속성 의사 결정방법은 유한개의 기준(Criteria)과 대안(Alternatives)들이 있을 때, 하나 혹은 몇 가지의 대안 을 선정하는 것으로 미리 정해진 범위 내에서 우선순위를 정하는 방식이다. $\mathrm{AHP}$ 는 의사결정의 목 표 또는 평가기준이 다수이며 복합적이어서 결정이 어려운 경우, 주요 기준을 세부 기준으로 분해 하고, 이들을 쌍대 비교(Pair-wise Comparison)를 통해 분석하는 방법이다. 이는 다수의 속성을 계 층으로 분류하여 분석하는데 효과적이며 주로 대규모 사업 분석 등에 활용된다. 군에서는 신규 무 기 체계 선정을 위한 제반 평가기준을 마련하거나 여러 가지 대안 중 가장 최적의 대안을 선정하는 등 다양한 의사결정 문제에 직면했을 때 전문가 의견을 반영하여 최대한 객관적이고도 정량적인 결과를 도출하고자 하는 경우에 주로 활용한다. $\mathrm{AHP}$ 기법의 대략적인 적용 절차는 크게 6 단계로 나뉜다(Table 1).

$<$ Table 1> Application procedure of AHP

\begin{tabular}{|c|c|}
\hline Step & Main contents \\
\hline Step 1 & Decomposition of given decision criteria, alternatives into hierarchical structure \\
\hline Step 2 & $\begin{array}{l}\text { Conducting a survey of decision elements in the same hierarchy for pairwise } \\
\text { comparisons }\end{array}$ \\
\hline Step 3 & $\begin{array}{l}\text { Estimating the relative importance or weight of the components compared with the } \\
\text { Eigenvalues method }\end{array}$ \\
\hline Step 4 & $\begin{array}{l}\text { Consistency verification by consistency analysis (if } \mathrm{CR} \text { values are less than } 0.1 \text { go } \\
\text { to Step } 6 \text {, otherwise go to Step 5) }\end{array}$ \\
\hline Step 5 & Reset initial values in the same way as in Step 2 and revert back to Step 3 \\
\hline Step 6 & $\begin{array}{l}\text { Summing the weights for each evaluation element calculated at each layer leads to } \\
\text { prioritization of alternative in the lowest layer }\end{array}$ \\
\hline
\end{tabular}




\section{2 가중치 도출}

$\mathrm{AHP}$ 기법에서는 일반적으로 9점 척도를 이용하여 기준 혹은 대안별 가중치를 계산한다. 쌍대 비교를 통해 기준 간 중요도가 비교행렬(Comparison Matrix)의 형태로 나타나게 되며, Table 2와 같이 표현할 수 있다.

$<$ Table 2> Comparison matrix

\begin{tabular}{c|c|c|c|c}
\hline & Criterion 1 & Criterion 2 & $\cdots$ & Criterion $n$ \\
\hline \hline Criterion 1 & 1 & $a_{12}$ & $\cdots$ & $a_{1 n}$ \\
\hline Criterion 2 & $a_{21}$ & 1 & $\cdots$ & $a_{2 n}$ \\
\hline$\vdots$ & $\vdots$ & $\vdots$ & 1 & $\vdots$ \\
\hline Criterion $n$ & $a_{n 1}$ & $a_{n 2}$ & $\cdots$ & 1 \\
\hline
\end{tabular}

비교행렬을 바탕으로 기준 $i$ 의 가중치 $\left(w_{i}\right)$ 는 식 (1)과 같이 산출한다.

$$
w_{i}=\frac{1}{n} \sum_{j \in J} \frac{a_{i j}}{T_{j}}, \forall i \in I
$$

여기서 $w_{i}$ 는 기준 $i$ 의 가중치, $n$ 은 기준의 개수, $a_{i j}$ 는 비교행렬의 $(i, j)$ 번째 요소, $T_{j}$ 는 비교행렬 의 $j$ 번째 열의 요소들의 총합을 나타낸다.

\section{3 일관성 분석}

$\mathrm{AHP}$ 기법은 가중치를 결정하는 과정에서 일관성 분석을 통해 결과의 유효성을 판단한다. 일관 성 비율 값인 $\mathrm{CR}$ (Consistence Ratio)이 0.1 이하일 경우 일관성이 있으며 유효하다고 판단한다. 한 편 $\mathrm{CR} \leq 0.2$ 일 경우는 비전문가 집단의 응답인 경우에 활용하며 용납 가능한 일관성을 갖는다. 단, 본 연구는 전문가 집단을 대상으로 설문조사를 진행하였으므로 $\mathrm{CR} \leq 0.1$ 을 활용한다. 일관성 비율 은 일관성 지수(CI: Consistency Index)를 무작위 지수(RI: Random consistency Index)로 나눈 값 이다. 먼저 일관성 지수의 계산은 비교행렬(Table 2 에 제시된 바와 같은 $n \times n$ 행렬)의 원리 고유치 (Principle Eigenvalue) $\lambda_{\max }$ 를 구한 후 식 (2)와 같이 도출한다.

$$
C I=\frac{\lambda_{\max }-n}{n-1}
$$


다음으로 무작위 지수는 Table 3에 나타나는 바와 같으며, $n$ 의 값에 따른 차이를 보인다. 무작위 지수는 $\operatorname{Satty}(1980)$ 가 임의로 비교행렬을 생성하여 약 500개의 표본공간에 대하여 구한 후 이를 표 로 제시한 것이다.

$<$ Table 3> Random Index

\begin{tabular}{c|c|c|c|c|c|c|c|c|c}
\hline$n$ & 1 & 2 & 3 & 4 & 5 & 6 & 7 & 8 & 9 \\
\hline \hline $\mathrm{RI}$ & 0.00 & 0.00 & 0.58 & 0.90 & 1.12 & 1.24 & 1.32 & 1.41 & 1.45 \\
\hline
\end{tabular}

마지막으로 일관성 비율은 $C R=C I / R I$ 에 따라 도출하며 이 값이 0.1 이하이면 일관성이 있는 것으로 판단한다.

\section{III. 무인잠수정 평가기준 선정}

일반적으로 무기체계 도입에는 다수의 전문가 집단의 집단의사결정 과정이 포함된다. 특히 신규 무기체계 선정을 위한 제반 평가기준을 마련할 때, 최초 전문가 인터뷰 및 의견을 수렴하여 기준을 선정하며 선정된 기준별 중요도를 설정하기 위하여 과학적 방법론을 활용한다. 먼저 평가기준 선정 을 위하여 선행 연구에서 제시된 연구결과를 통해 UUV의 특성을 고려하였으며, 이를 바탕으로 잠 수함 관련 전문가 인터뷰를 통하여 감시정찰용 UUV에 요구되는 특성을 요약 및 정리하였다.

다음으로, 선정된 평가기준에 대한 중요도 설정을 위하여 $\mathrm{AHP}$ 기법을 활용하였으며, $\mathrm{AHP}$ 는 다 수의 전문가들의 설문조사, 의견을 기반으로 기준, 계층, 가중치가 설정되는 점에서 집단의사결정 을 바탕으로 최대한 객관적인 기준을 마련한다는데 그 장점이 있다. 또한 $\mathrm{AHP}$ 는 기준 선정에 있어 일관성을 분석하여 그 유효함을 판단함으로써, 주관적이고 신뢰할 수 없는 자료를 제거하여 연구의 신뢰성을 높여줄 수 있기 때문에 신규 UUV 개발을 위한 기준 설정에 적절하다고 판단하였다.

\section{1 전문가 자문 및 설문조사}

본 연구에서는 평가기준 선정을 위한 전문가 자문을 1 회 실시하였으며, 선정된 평가기준별 중요 도 측정을 위한 $\mathrm{AHP}$ 설문을 1회 실시함으로써 총 2회의 과정을 통해 평가기준을 수립하고 이를 분석하였다. 감시정찰용 UUV의 요구성능 분석이라는 연구 주제에 적합하도록 실제 잠수함에 근무 하였거나 운용해 본 경험이 있는 해군 장교 위주로 자문 및 설문조사를 수행하였다.

먼저 최초 평가기준 선정을 위한 전문가 자문은 잠수함에서 주요 보직을 수행한 경험이 있는 장 교 5명을 선정하여 2020년 3월 중 실시하였다. 인터뷰는 개별방문을 통해 진행하였으며, 이들은 잠 
수함에서 지휘관 또는 부서장으로 근무한 경험을 바탕으로 미래에 운용하게 될 감시정찰 목적의 $\mathrm{UUV}$ 에게 요구되는 성능을 개념적으로 제시하고 그에 요구되는 성능 또한 제시하였다. 인터뷰 결 과 나타난 광범위하고도 다양한 의견들 중 공통적으로 언급된 내용을 바탕으로 각각의 적절성에 대한 검토를 통해 최종적으로 UUV에 요구되는 특성을 크게 5가지로 분류하였으며, 이를 대기준으 로 정의하였다. 또한, 각각의 대기준의 개념과 고려사항을 검토하여 3 가지의 하위 기준을 도출하였 으며, 이를 소기준으로 분류하였다.

다음으로 선정된 평가기준에 대한 중요도(가중치)를 도출하기 위해 $\mathrm{AHP}$ 기법을 적용하였다. 2 장에서 설명된 바와 같이, $\mathrm{AHP}$ 기법은 동일한 계층 내에 존재하는 기준들에 대해 각각 쌍대비교를 실시하여 가중치를 계산하는 방법이며, 기준 간 쌍대비교는 전문가들로부터의 의견을 바탕으로 수 행된다. 가중치 도출을 위한 설문조사는 총 51 명을 대상으로 실시하였으며, 집단의 구성은 잠수함 근무 경험을 보유한 잠수함 장교 47명과 관련 연구 등을 수행한 학계 전문가 4명으로 구성하였다.

\section{2 대기준 선정 결과}

대기준은 미 해군의 '2004 UUV Master Plan(US Navy, 2004)'이 제시하는 감시 - 정찰(ISR: Intelligence, Surveillance and Reconnaissance) 임무 수행에 필요한 기술을 참고하였다. UUV가 감 시 - 정찰 임무를 수행하기 위해서는 UUV의 작전지속성, 광범위한 통신능력, 물체를 회피하여 기 동하는 능력, 은밀한 작전 능력이 필요하다. 이와 더불어 무기체계 도입에서 비용 측면을 간과할 수 없는데, 비용에 대한 고려가 결여된 무기체계의 도입은 현실성과 타당성이 떨어지기 때문이다. 따라서 본 연구에서는 통신능력, 지속성, 은밀성, 기동성, 경제성을 UUV 개발 시 고려되어야 하는 대기준으로 선정하였으며, 각각에 대한 세부내용은 다음과 같다.

첫째, 통신능력은 UUV와 아군 간의 정보 송 - 수신이 가능한 능력이다. 감시 - 정찰 임무를 수행 하는 UUV는 수집한 정보(적 잠수함이 모항을 출항하였다는 정보 등)를 아군 모함 혹은 부대에 전 송하여 적군의 상황을 파악할 수 있게 해야 한다. 따라서 통신능력은 UUV에 필수적인 능력이다. 둘째, 지속성은 UUV가 작전 수행이 가능한 시간과 관련된 능력이다. 모함 혹은 항구에서 출항한 $\mathrm{UUV}$ 는 장시간 항해하며 적군의 동태를 감시 - 정찰해야 한다. 이를 위해선 UUV의 작전 지속성이 중요하다. 셋째, 은밀성은 UUV가 적군에게 탐지되지 않고 임무수행을 할 수 있는 능력이다. 감시 정찰 임무를 수행하는 UUV는 적에게 발각되지 않고 안전하게 임무를 진행해야 한다. 넷째, 기동성 은 UUV가 임무수행 시 지형지물의 영향을 최소한으로 받으며 기동하는 능력이다. UUV는 자율주 행을 기반으로 운용되기 때문에 스스로 장애물을 감지, 회피하여 기동하는 능력이 필수적이다. 다 섯째, 경제성은 UUV 건조 및 운용유지 비용 대비 효율성을 의미한다. 예를 들어 $\mathrm{A}, \mathrm{B}$ 라는 두 종류 의 UUV가 도입할 후보라고 할 경우, 똑같은 성능을 보유했다고 가정했을 때 비용이 더욱 저렴한 것을 선호하게 될 것이며, 이러한 경제성이 고려되어야 함을 의미한다. 앞서 언급한 것과 같이 무기 
체계 도입 시 무기체계 도입 및 운용유지 비용과 이를 운용할 수 있는 기간에 대한 고려는 필수적 이다.

\section{3 소기준 선정 결과}

먼저 통신능력의 하위 기준으로써 위성통신, 부이통신, 수중통신 등 3 가지의 소기준을 선정하였 다. 위성통신은 UUV가 위성을 매개로 하여 모함 혹은 부대와 통신하는 것으로, UUV에 위성 통신 안테나의 장착이 요구된다. 부이통신은 해수면에 설치된 통신 부이를 매개로 통신하는 것으로, 해 수면에 통신 능력이 장착된 여러 개의 부이를 설치해야 한다. 수중통신은 UUV가 수면 위로 부상 하지 않고도 잠항 중에 수중에서 정보를 송·수신하는 것을 의미한다.

지속성의 하위 기준으로 동력원, 연비, 내구성 등 3 가지의 소기준을 선정하였다. 동력원은 UUV 를 구동하는 에너지의 종류로서 장시간 수중에서 운용하며 여러 장비를 구동하기 위해선 그에 적 합한 고농축의 에너지가 요구된다. 연비는 연료의 효율성을 의미하는 것으로, UUV가 장시간 기동 하며 임무를 수행하기 위해선 적은 양의 에너지로 최대한 많은 거리를 항해할 수 있어야 한다. 내 구성은 UUV가 내 - 외부의 변형 없이 견디는 정도를 나타내며, 결함 또는 충격에 대한 강한 내구 성이 요구된다.

은밀성에는 피탐, 소음, 재질 등 3가지의 소기준을 선정하였다. 먼저 피탐과 소음은 UUV가 임무 수행 중 적의 탐지를 회피하는데 필수적인 요소이며, 재질 또한 적 탐지를 회피하기에 효과적인 금 속 혹은 도료를 사용해야 한다.

기동성에는 속도, 위험물 회피 능력, 심도 유지 등 3가지의 소기준을 선정하였다. UUV가 효과적 인 감시·정찰 임무 수행을 위해서는 요구되는 능력의 속도가 필요하며, 위험물을 스스로 회피하 여 기동할 수 있는 능력 또한 요구된다. 또한 UUV는 유인잠수함에 비해 크기가 작고 자율주행으 로 운용되므로 수압과 물살을 견디며 심도를 유지하는 능력 또한 요구된다고 할 수 있다.

마지막으로 경제성의 하위 기준은 운용기간, 건조비용, 유지비용 등 3 가지의 소기준로 선정하였 다. 이는 총수명주기비용과 시스템 엔지니어링 개념을 반영한 결과로서 무기체계의 도입뿐만 아니 라 운용유지에 소요되는 전체 비용을 고려하여야 함을 반영한 결과이다.

Table 4는 지금까지 제시된 대기준 및 소기준을 요약하여 제시한 결과이며, 이를 AHP 분석을 위해 계층화된 형태로 나타내면 Figure 1과 같다. 
$<$ Table $4>$ Results of selection of assessment criteria for UUV

\begin{tabular}{|c|c|c|}
\hline Criteria & Sub Criteria & Definition \\
\hline \multirow{3}{*}{$\begin{array}{l}\text { Communication } \\
\text { Capability }\end{array}$} & $\begin{array}{c}\text { Satellite } \\
\text { communication }\end{array}$ & $\begin{array}{l}\text { Sending and receiving information with UUV and allies using } \\
\text { satellites }\end{array}$ \\
\hline & $\begin{array}{c}\text { Buoy } \\
\text { Communication }\end{array}$ & $\begin{array}{l}\text { Sending and receiving information by means of a buoy installed } \\
\text { on the sea level }\end{array}$ \\
\hline & $\begin{array}{l}\text { Underwater } \\
\text { Communication }\end{array}$ & $\begin{array}{l}\text { UUV sends and receives information underwater while } \\
\text { underwater }\end{array}$ \\
\hline \multirow{3}{*}{ Persistence } & Power Source & The type of energy that drives the UUV \\
\hline & Fuel efficiency & Amount of fuel consumed by the UUV per hour \\
\hline & Durability & $\begin{array}{l}\text { The degree to which the UUV withstand internal and external } \\
\text { deformation without any internal or external deformation }\end{array}$ \\
\hline \multirow{3}{*}{ Stealthiness } & Avoid Detection & The ability to evade enemy detection \\
\hline & Noise & Degree of noise generated by UUV \\
\hline & $\begin{array}{c}\text { The properties of a } \\
\text { material }\end{array}$ & Properties of Materials that make up the UUV Surface \\
\hline \multirow{3}{*}{ Mobility } & Speed & Speed of UUV maneuver \\
\hline & $\begin{array}{l}\text { Ability to avoid } \\
\text { dangerous goods }\end{array}$ & $\begin{array}{c}\text { Ability to maneuver by avoiding obstacles and dangerous } \\
\text { objects during UUV manoeuvres }\end{array}$ \\
\hline & Keep in Depth & $\begin{array}{l}\text { The ability of the UUV to maintain its depth during the } \\
\text { incubation period }\end{array}$ \\
\hline \multirow{3}{*}{$\begin{array}{l}\text { Economic } \\
\text { Efficiency }\end{array}$} & Operation period & Duration of operation in the field after manufacturing the UUV \\
\hline & Cost of Construction & Costs Required for UUV Construction \\
\hline & Cost of Maintenance & Costs Required for UUV Maintenance \\
\hline
\end{tabular}

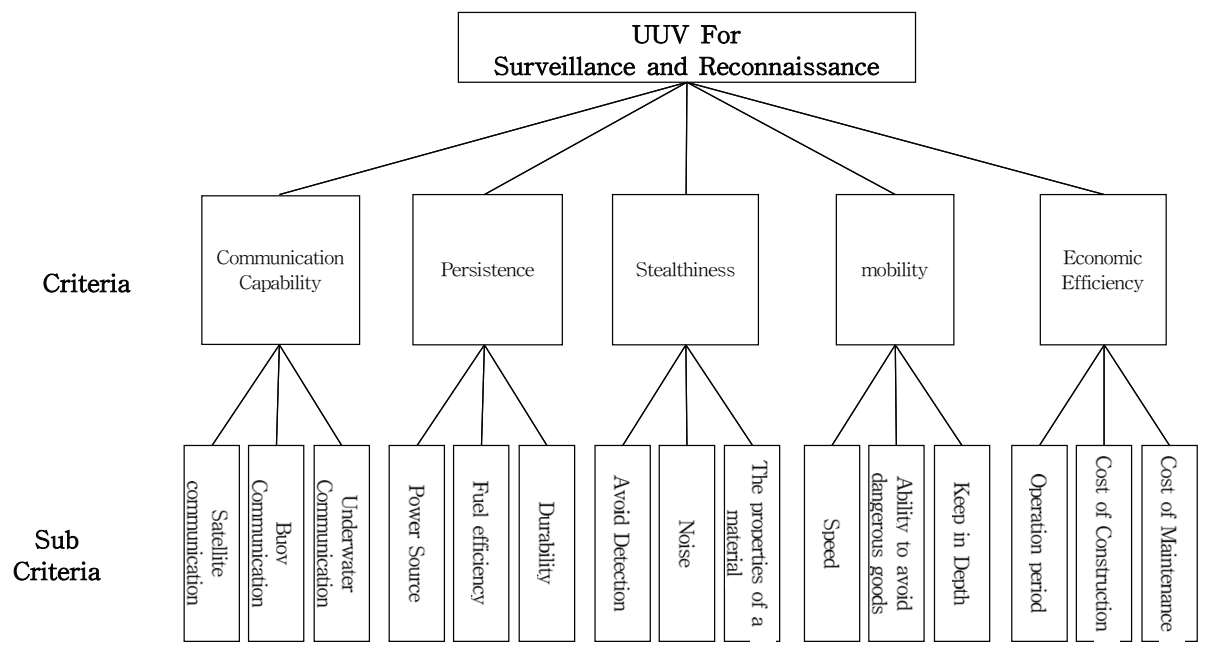

$<$ Figure 1> Hierarchical Structure of UUV Assessment Criteria 
Prioritizing the capabilities of unmanned ... / Gwuihyeon Park $\cdot$ Taehyun Lee $\cdot$ Chunghwan Lee $\cdot$ Jinho Lee 11

\section{AHP 분석 결과}

\section{1 일관성 비율 검증}

$\mathrm{AHP}$ 기법의 가장 큰 특징은 여러 기준들 간에 쌍대비교 결과를 이용하여 식 (1)과 각각의 기준 에 대한 가중치를 도출하는 것이다. 그러나 이를 실행하기 이전 단계에서 우선적으로 선행되어야 하는 것이 일관성 검증이다. 즉 설문 참여자가 답변한 결과에 일관성을 가려냄으로서 일관성이 없 다고 판단된 결과가 가중치를 도출하는데 반영되지 않도록 해야 한다. 2.3절에 언급된 바와 같이 일관성 검증은 일관성 비율 $(\mathrm{CR})$ 이 0.1 이하일 때 설문 결과가 일관성이 있다고 판단하며, 일관성 비율은 식 (2)의 일관성 지수(CI)와 Table 3의 무작위 지수(RI)의 비율로 나타낸다.

설문은 총 51 명의 잠수함 또는 해당 분야 전문가에 대하여 실시하였으며, 이 중 24 명의 결과는 일관성 검증을 통과하지 못하여 분석 자료로는 적절치 않다고 판단하여 제외하였다. 따라서 일관성 검증을 통과한 총 27 명의 설문 결과에 대하여 가중치 도출을 위한 분석을 수행하였다. 본 연구에서 는 설문 결과 중 대기준 간 일관성 검증이 통과된 경우, 특정 대기준 내 소기준 간 일관성 검증이 통과된 경우에 대해서는 해당 기준 간 가중치를 도출하는 곳에는 사용되었다.

총 27 개의 일관성 검증을 통과한 설문 결과 중 대기준을 통과한 설문은 10 개, 소기준 1 은 12 개, 소기준 2 는 18 개, 소기준 3 은 17 개, 소기준 4 는 15 개, 소기준 5 는 22 개였으며, 이를 요약한 결과는 Table 5와 같다.

$<$ Table 5> Result of verifying consistency ratio per criteria

\begin{tabular}{|c|c|c|c|c|c|c|c|}
\hline \multirow{3}{*}{ Division } & $\begin{array}{c}\text { Evaluation } \\
\text { Target }\end{array}$ & Criteria & $\begin{array}{c}\text { Sub Criteria } \\
1\end{array}$ & $\begin{array}{c}\text { Sub Criteria } \\
2\end{array}$ & \begin{tabular}{|c|} 
Sub Criteria \\
3
\end{tabular} & $\begin{array}{c}\text { Sub Criteria } \\
4\end{array}$ & $\begin{array}{c}\text { Sub Criteria } \\
5\end{array}$ \\
\hline & Factors & 5 & 3 & 3 & 3 & 3 & 3 \\
\hline & $\begin{array}{c}\text { Evaluation } \\
\text { Method } \\
\end{array}$ & $\begin{array}{c}\text { Pairwise } \\
\text { Comparison } \\
\end{array}$ & $\begin{array}{c}\text { Pairwise } \\
\text { Comparison } \\
\end{array}$ & $\begin{array}{c}\text { Pairwise } \\
\text { Comparison } \\
\end{array}$ & \begin{tabular}{|c|} 
Pairwise \\
Comparison \\
\end{tabular} & \begin{tabular}{c|} 
Pairwise \\
Comparison \\
\end{tabular} & $\begin{array}{c}\text { Pairwise } \\
\text { Comparison } \\
\end{array}$ \\
\hline \multirow{12}{*}{ Evaluator } & 1 & 0.1476 & -0.0002 & 0.0448 & -0.0002 & 0.0146 & -0.0002 \\
\hline & 2 & 0.3681 & 0.4012 & 0.0960 & 0.0092 & 0.3057 & 0.0835 \\
\hline & 3 & 0.9422 & 0.4012 & -0.0002 & 1.2901 & -0.0002 & 0.0473 \\
\hline & 4 & 0.0022 & -0.0002 & 0.1524 & 0.0003 & 0.0003 & 0.0003 \\
\hline & 5 & 0.2321 & 0.0146 & 0.6721 & -0.0004 & 0.0037 & -0.0002 \\
\hline & 6 & 0.0719 & 0.3405 & 0.0005 & 0.0960 & 0.3405 & 0.0960 \\
\hline & 7 & 0.3700 & 0.8492 & 0.4164 & 0.0005 & 0.6502 & -0.0002 \\
\hline & 8 & 0.6571 & 0.9517 & 0.6805 & -0.0002 & 0.0628 & 0.3061 \\
\hline & 9 & 0.1159 & -0.0001 & 0.3061 & -0.0002 & -0.0002 & -0.0002 \\
\hline & 10 & 0.1572 & 0.2638 & 0.4012 & 0.0005 & 0.0191 & 0.2638 \\
\hline & 11 & 0.7835 & 0.2403 & 0.0005 & 0.4012 & 0.0960 & -0.0002 \\
\hline & 12 & 0.7195 & 0.9517 & -0.0001 & -0.0004 & -0.0001 & -0.0002 \\
\hline
\end{tabular}




\begin{tabular}{|c|c|c|c|c|c|c|}
\hline 13 & 0.5242 & 0.0191 & 0.0191 & 0.2801 & 0.0146 & -0.0002 \\
\hline 14 & 0.0479 & 0.1277 & 0.0061 & 0.1524 & 0.1524 & -0.0002 \\
\hline 15 & 0.0001 & 0.6721 & -0.0001 & 0.0005 & -0.0002 & 0.0005 \\
\hline 16 & 0.1624 & 0.5169 & 0.4644 & 0.6721 & 0.1639 & -0.0002 \\
\hline 17 & 0.0512 & 0.0960 & 0.0960 & -0.0002 & 0.0473 & 0.0473 \\
\hline 18 & 0.5418 & -0.0001 & 0.4644 & 0.6721 & 0.5253 & 0.8865 \\
\hline 19 & 0.4717 & 0.2180 & 0.0960 & 0.6721 & 0.9633 & 0.4164 \\
\hline 20 & 0.1028 & 0.4012 & 0.0003 & -0.0002 & 0.1524 & -0.0002 \\
\hline 21 & 1.2415 & 2.0594 & 0.0312 & 0.8688 & 0.1894 & 0.1894 \\
\hline 22 & 0.0485 & -0.0002 & 0.0473 & 0.5253 & 0.1894 & 0.0960 \\
\hline 23 & 0.0606 & 0.0312 & 0.4644 & 0.6721 & 0.2638 & -0.0002 \\
\hline 24 & 0.1024 & 0.7511 & -0.0002 & -0.0002 & 0.5258 & -0.0002 \\
\hline 25 & 0.0769 & 0.0473 & -0.0002 & -0.0002 & 0.0003 & 0.0234 \\
\hline 26 & 0.0038 & 0.0096 & 0.0096 & 0 & 0.0096 & 0 \\
\hline 27 & 0.0138 & 0.0473 & 0 & -0.0002 & 0 & -0.0002 \\
\hline \# Passes & 10 & 12 & 18 & 17 & 15 & 22 \\
\hline
\end{tabular}

\section{2 계층별 분석 결과}

일관성 검증을 통과한 설문결과에 대하여 식 (1)의 가중치(중요도)를 도출하였다. 먼저 대기준의 문항별 중요도를 분석한 결과, 통신능력의 중요도가 0.3406으로 가장 높게 나타났으며 은밀성과 기 동성이 각각 0.2764 및 0.1609 로 2,3 위의 우선순위를 차지하였다. 마지막으로 지속성과 경제성은 각각 $0.1477,0.0745$ 로서 가장 낮은 우선순위를 보여주었다(Table 6). 이를 바탕으로 잠수함 또는 해 당 분야의 전문가 의견을 종합해 보면, 감시 - 정찰 임무를 수행해야 하는 UUV에게 통신능력이 가 장 중요하게 요구됨을 알 수 있으며, 은밀성과 기동성이 차순위로 중요하게 고려되고 있음을 알 수 있다. 이에 반해 지속성과 경제성의 경우에는 다른 요구성능에 비해서는 상대적으로 중요도가 낮다 고 평가되었는데, 이는 국가 안보와 관련된 군 임무의 특성상 비용보다는 성능을 우선시하는 경향 이 반영된 것으로 해석된다.

다음으로 각각의 대기준 내에서 선정된 3개의 소기준을 대상으로 동일한 방식으로 상대적 중요 도를 도출하였으며 그 결과는 Table 6의 소기준에 대한 중요도에 나타나는 바와 같다. 따라서 각각 의 대기준 내에서 3 개씩의 소기준 간 상대적 중요도를 확인할 수 있으며, 특히 중요도가 가장 높은 통신능력의 경우 위성통신, 부이통신, 수중통신 순으로 높은 우선순위를 차지하였다. 또한, 소기준 전체 15 개에 대한 상대적 중요도를 관찰해 보면, 지속성의 하위기준인 내구성의 중요도가 0.5071 로 가장 높게 나타났으며 다음으로 통신능력의 하위기준인 위성통신(0.4845), 은밀성의 하위기준인 피 탐(0.4398) 순으로 나타났다. 
$<$ Table 6> Result of relative importance per criteria

\begin{tabular}{|c|c|c|c|}
\hline $\begin{array}{c}\text { Criteria } \\
\text { (Importance) }\end{array}$ & Sub Criteria & Importance & Priority \\
\hline \multirow{3}{*}{$\begin{array}{c}\text { Communication } \\
\text { Capability } \\
(0.3406)\end{array}$} & Satellite Communication & 0.4845 & 1 \\
\hline & Buoy Communication & 0.2837 & 2 \\
\hline & Underwater Communication & 0.2319 & 3 \\
\hline \multirow{3}{*}{$\begin{array}{c}\text { Stealthiness } \\
(0.2764)\end{array}$} & Avoid Detection & 0.4398 & 1 \\
\hline & Noise & 0.3771 & 2 \\
\hline & The properties of a material & 0.1830 & 3 \\
\hline \multirow{3}{*}{$\begin{array}{l}\text { Mobility } \\
(0.1609)\end{array}$} & Ability to avoid dangerous goods & 0.4085 & 1 \\
\hline & Keep in Depth & 0.3568 & 2 \\
\hline & Speed & 0.2348 & 3 \\
\hline \multirow{3}{*}{$\begin{array}{c}\text { Persistence } \\
(0.1477)\end{array}$} & Durability & 0.5071 & 1 \\
\hline & Power Source & 0.3134 & 2 \\
\hline & Fuel Efficiency & 0.1795 & 3 \\
\hline \multirow{3}{*}{$\begin{array}{c}\text { Economic } \\
\text { Efficiency } \\
(0.0745)\end{array}$} & Operation period & 0.3931 & 1 \\
\hline & Cost of Maintenance & 0.3570 & 2 \\
\hline & Cost of Construction & 0.2524 & 3 \\
\hline
\end{tabular}

$<$ Table $7>$ Result of complex importance per sub criteria

\begin{tabular}{|c|c|c|c|}
\hline Criteria & Sub Criteria & $\begin{array}{c}\text { Complex } \\
\text { Importance } \\
\end{array}$ & Priority \\
\hline \multirow{3}{*}{$\begin{array}{l}\text { Communication } \\
\text { Capability }\end{array}$} & Satellite Communication & 0.1650 & 1 \\
\hline & Underwater Communication & 0.0790 & 5 \\
\hline & Buoy Communication & 0.0966 & 4 \\
\hline \multirow{3}{*}{ Persistence } & Power Source & 0.0463 & 10 \\
\hline & Fuel Efficiency & 0.0265 & 14 \\
\hline & Durability & 0.0749 & 6 \\
\hline \multirow{3}{*}{ Stealthiness } & Avoid Detection & 0.1216 & 2 \\
\hline & Noise & 0.1042 & 3 \\
\hline & The properties of a material & 0.0506 & 9 \\
\hline \multirow{3}{*}{ Mobility } & Speed & 0.0378 & 11 \\
\hline & Ability to avoid dangerous goods & 0.0657 & 7 \\
\hline & Keep in Depth & 0.0574 & 8 \\
\hline \multirow{3}{*}{$\begin{array}{l}\text { Economic } \\
\text { Efficiency }\end{array}$} & Operation period & 0.0293 & 12 \\
\hline & Cost of Construction & 0.0188 & 15 \\
\hline & Cost of Maintenance & 0.0266 & 13 \\
\hline
\end{tabular}


그러나 상위기준이 각각 다른 상태에서 15 개의 소기준을 동일한 선상에서 비교하는 것은 큰 의 미가 없으며, 이를 동일선상에서 비교하기 위해서는 대기준과 소기준의 중요도를 곱한 값인 복합 중요도를 관찰해 보아야 한다. Table 7은 각각의 소기준에 대한 복합 중요도를 계산한 결과와 그에 따른 우선순위를 보여준다. 위성통신이 $0.1650(=0.3406 \times 0.4845)$ 으로 복합중요도가 가장 높았다. 다 음으로 피탐이 0.1216으로 두 번째로 높았고, 소음이 0.1042로 세 번째로 높았다. 즉, 위성통신과 피 탐의 경우 소기준 중요도에 비해 복합 중요도 분석에서의 순위가 상승한 반면, 소기준 중 중요도가 가장 높았던 내구성의 경우에는 복합중요도 0.0749 로 하락한 것은 상위기준의 중요도가 반영된 결 과라 할 수 있다. 복합 중요도를 기준으로 볼 때 통신, 피탐, 소음 등 UUV의 성능적 측면에 대한 중요성은 높게 인식하는 반면 경제적 측면에서의 지표는 상대적으로 낮게 나타남을 알 수 있다.

\section{$\mathrm{V}$. 결론 및 논의}

본 연구의 목적은 감시 - 정찰용 UUV가 갖추어야 할 구체적인 특성 및 요구성능을 분석하고 그 에 관한 우선순위를 도출하는 데 있다. 이를 위해 잠수함 또는 국방 분야에 종사하는 전문가 집단 을 구성하여 면담 및 설문조사를 실시하여 자료 분석의 객관성과 전문성을 확보하였다. 그 결과 5 가지의 대기준과 각각의 대기준별 3 개씩 총 15 개의 소기준을 마련하였다. 다음으로 각각의 기준에 대한 상대적 중요도와 우선순위를 도출하기 위하여 쌍대비교 기반의 설문을 실시한 후 AHP 기법 을 이용하였다. 분석 결과, 5 가지의 대기준에 관한 중요도를 도출할 수 있었으며 대기준별 3 가지로 구성된 소기준에 대한 중요도 또한 제시하였다. 추가적으로 하위개념에 해당하는 소기준 15 개를 동 일한 기준으로 비교해보기 위하여 복합 중요도를 관찰하였다. 전문가 집단의 의견을 종합적으로 비 추어 볼 때, 전반적으로 감시 - 정찰에 요구되는 UUV의 성능 및 능력에 관한 중요도가 매우 높게 나타났으며, UUV의 운용적 측면에서 고려되는 비용, 경제성, 효율성 등의 중요도는 상대적으로 낮 게 나타났다. 이러한 결과는 향후 감시 - 정찰 임무를 수행하게 될 UUV를 실제 개발하는 과정에서 참고자료가 될 수 있을 뿐만 아니라, 다른 임무를 수행하게 될 UUV의 개발 시 요구성능 도출에 유용하게 활용될 것으로 기대된다.

본 연구에서 제시한 감시 - 정찰 목적의 UUV의 요구성능은 기술적 부분이 아닌 개념적인 요구 성능을 의미한다. 즉 개발될 새로운 무기체계가 어떠한 능력을 우선적으로 보유할 것인가에 대한 논의이다. 따라서 이는 신형 무기체계의 개발에 필요한 개념적 요구사항의 정립에 참고로 활용될 수 있는 결과이며, 향후 기술적 가능성과 보다 구체화된 플랫폼의 요구사항을 제시하는데 상위개념 의 근거가 될 수 있을 것이다. 또한 본 연구에서 제시하는 기준별 중요도(가중치)는 향후 선택 가능 한 UUV 후보들이 존재하게 되면 각각의 후보를 정량적으로 평가하는 기준으로도 활용이 가능하 다. 또한 연구 방법론적 측면에서 볼 때, 새로운 기준의 정립을 위해 수행된 전문가 자문은 주관적 
인 판단의 개입이 불가피한 측면이 있다. 그러나 기존의 기준이나 척도가 존재하지 않을 때 최초 단계에서 전문가 자문을 통한 아이디어의 도출은 일반적으로 행해지는 연구 방법이며, 기준이 정립 된 이후에는 $\mathrm{AHP}$ 기법과 같은 객관적인 가중치 도출 방법을 적용함으로서 최대한 객관화하려는 시도를 보였다는 점에서 그 의의가 있다. 이러한 방법은 향후 기타 신형 무기체계, 특히 최근 발전 을 거듭하고 있는 무인체계에도 그 적용 가능성을 확대할 수 있음을 시사한다.

본 연구에서 최대한 객관적이고도 구체적인 요구성능 분석을 위해 전문가 집단 및 $\mathrm{AHP}$ 기법 등 을 활용하였음에도 불구하고 몇 가지 제한점 및 향후 보완되어야 할 사항 또한 존재한다. 먼저 전 문가 집단의 구성을 더욱 다양화할 필요성이 있다. 본 연구에서는 주로 해군 내 잠수함 근무 장교 등의 의견을 반영하였는데, 향후에는 방산업체, 조선소, 국방 관련 연구기관 등의 범위로까지 확대 된 의견의 수렴이 요구된다. 이는 운용적 측면에서의 요구성능으로 편중될 우려를 해소하고, UUV 의 제작 및 설계 측면에서의 고려사항을 함께 균형적으로 반영하기 위함이다. 또한, 전문가 집단의 구성을 다양화함에 따라 그 의견 또한 다양해질 것으로 기대된다. 보다 다양하고 방대해지는 의견 으로부터 핵심적인 내용을 추출하거나 이를 추정하기 위하여 빅데이터 분석 기법을 적용해 볼 수 있을 것이다.

다음으로 해군이 현재 보유하고 있는 전력과의 연계성을 고려한 연구가 필요하다. 본 연구에서 는 감시 - 정찰 UUV가 어떤 특성을 가져야 하는가를 제시하는 것에 집중하였다. 그러나 대부분의 전력은 단독적으로 임무를 수행하기 보다는 타 전력과 협동, 합동 운용(예를 들어, 무인잠수정과 유 인잠수정의 협동 또는 무인잠수정과 유인수상정 또는 대잠항공기의 협동 등)하므로 향후에는 전력 들과의 연계성, 상호운용성 등에 관한 부분을 함께 고려한 상황에서의 특성과 요구성능 도출이 이 루어진다면 보다 현실적인 방안이 될 것으로 기대된다.

\section{Acknowledgements}

We would like to thank Editage (www.editage.co.kr) for English language editing.

\section{Declaration of Conflicting Interests}

The author(s) declared no potential conflicts of interest with respect to the research, authorship, and/or publication of this article. 


\section{Reference}

Allard, Y., \& Shahbazian, E. (2014). Unmanned Undersea Vehicles (UUV) Information Study. OODA Technologies, Canada. https://apps.dtic.mil/sti/citations/AD1004191

Button, R. W., Kamp, J., Curtin, T. B., \& Dryden, J. (2009). A Survey of Missions for Unmanned Undersea Vehicles. RAND National Defense Research Institute, USA. https://apps.dtic.mil/sti/citations/ADA503362

Choi, J.-R., Kim, C., \& Kim, D. (2012). A trend of developing military purpose UUVs. Defense and Technology, 396, 52-69. https://www.dbpia.co.kr/journal/articleDetail?nodeId=NODE02304189

Hwang, A., Kim, M., Lee, S. Y., Yoon, J., \& Kim, C. (2011). A study on unmanned underwater vehicle operational performance analysis for mine search operations. Journal of the Korea Institute of Military Science and Technology, 14(5), 781-787. https://doi.org/10.9766/ KIMST.2011.14.5.781

Kang, S., Hong, S., \& Sur. J. (2019). A study on the operation scenarios of unmanned underwater vehicles for an ASW-ISR. Proceedings of the Conference of the Korea Institute of Military Science and Technology, 2239-2240

Lee, J. E. (2019). Technology development trends and development of unmanned underwater vehicle. Journal of Korea Academia-Industrial Cooperation Society, 20(9), 233-239. https://doi.org/10.5762/KAIS.2019.20.9.233

Lee, K., \& Yoon, H. (2011). Improvement of position estimation based on the multisensor fusion in underwater unmanned vehicles. Journal of Korean Institute of Intelligent Systems, 21(2), 178-185. UCI : G704-000319.2011.21..002

Lee, O.-S. (2017). A direction of development of large UUVs for anti-submarine warfare connected to the future warfare development trend of the Navy. Defense and Technology, 457, 80-89. http://www.dbpia.co.kr/journal/articleDetail?nodeId=NODE07118186

Lee, P.-M. (2020). A technology development trend and direction of Underwater drons, UUVs. Bulletin of the Society of Naval Architectures of Korea, 57(3), 2-6. https://www.dbpia.co.kr/journal/articleDetail?nodeId=NODE10442529

Satty, T. L. (1980). The Analytic Hierarchy Process: Planning, Priority Setting, Resource Allocation.McGraw-Hill.https://books.google.com/books/about/The_Analytic_Hierarchy_P rocess.html?id=Xxi7AAAAIAAJ

Shin, S., Lee, J., Hong, W., Kim, W. S., Bae, H., \& Cho, H. (2020). A simulator development for 
determining the sonar sensor configuration of unmanned underwater vehicles based on a hold-at-risk scenario. Journal of the Korea Society for Simulation, 29(2), 21-33. https://doi.org/10.9709/JKSS.2020.29.2.00221

US Navy (2004). The Navy Unmanned Undersea Vehicle (UUV) Master Plan. Department of the Navy, USA. https://www.hsdl.org/?view\&did=708654

Yao, H., Wang, H., Li, Y., Wang, Y., \& Han, C. (2019). Research on Unmanned Underwater Vehicle Threat Assessment, IEEE Access, 7, 11387-11396. https://doi.org/10.1109/ access.2019.2891940

Yoo, T.-S., Kim, M. H., Hwang, J. H., \& Yoon, S. L. (2016). Development of navigation HILS system for integrated navigation performance analysis of large diameter unmanned underwater vehicle. Journal of Ocean Engineering and Technology, 30(5), 367-373. https://doi.org/10.5574/KSOE.2016.30.5.367 
국문초록

\title{
$\mathrm{AHP}$ 를 이용한 감시정찰용 무인잠수정 요구성능 평가기준 분석
}

\author{
박귀현* • 이태현** 이충환*** 이진호****
}

현재 한반도의 안보상황에서 무인잠수정의 필요성은 점차 부각되고 있으며, 군사 분야의 무인잠수정이 수 행할 핵심임무로서 감시 및 정찰, 대기뢰전, 대잠전, 조사 및 식별, 해양탐사 등 5 가지가 제시되고 있다. 본 연 구에서는 무인잠수정의 핵심 임무 중에서도 가장 중요도가 높은 것으로 평가되는 감시 및 정찰 임무를 수행함 에 있어 우선적으로 고려되어야 할 특성 및 요구성능에 대한 평가기준을 분석한다. 먼저 해당분야 전문가의 자문을 통해 5 가지의 대기준과 15 가지의 소기준을 정립하였다. 다음으로 정립된 평가기준에 대한 상대적 중요 도와 우선순위를 도출하기 위하여 쌍대비교를 통해 가중치를 도출하는 AHP 기법을 활용하였으며, 별도의 전 문가 집단 51 명으로부터 쌍대비교 설문조사를 수행하였다. 분석결과, 감시 및 정찰에 요구되는 무인잠수정의 성능과 능력에 관한 중요도는 매우 높게 나타난 반면, 운용적 측면에서 고려되는 비용 및 경제성 등의 중요도 는 상대적으로 낮게 나타났다. 이러한 결과는 향후 무인잠수정 도입 시, 보다 객관적이며 구체적인 요구성능 에 대한 평가기준을 마련하는데 유용하게 활용될 것으로 기대된다.

주제어 : 분석적 계층화 과정(AHP), 무인잠수정, 정보감시정찰, 요구성능

*(제1저자) 해군사관학교, 국방경영학과, 학사과정, asa975@naver.com

** (공동저자) 해군사관학교, 국방경영학과, 학사과정, taelee456@gmail.com

*** (공동저자) 해군사관학교, 국방경영학과, 학사과정, leech3348@naver.com **** (교신저자), 해군사관학교, 국방경영학과, 교수, jinho7956@gmail.com 